\title{
LE RIAD: D'UN LIEU DE VILLÉGIATURE À UN LIEU DE MÉMOIRE DANS LA VIEILLE DAME DU RIAD DE FOUAD $\underline{\text { LAROUI }}$
}

Moufida El Bejaoui, Université Mohammed V, Faculté des Sciences de l'Éducation, Rabat, Maroc, moufidael@yahoo.fr

10.31902/fll.29.2019.9

UDK 821.133.1(64).09-31

Résumé: François et Cécile s'achètent un riad à Marrakech. A leur grande stupéfaction, ils y découvrent Massouda qui exige, pour vider les lieux, le retour de son fils Tayeb. Le couple sollicite l'aide du voisin Mansour. Celui-ci leur remet un mémoire dans lequel la vie du fils de Massouda recouvre un large pan de l'histoire commune du Maroc et de la France allant du Protectorat à la deuxième guerre mondiale.

Mots-clés: Fiction, Histoire, Colonialisme, Satire, Écriture, Mémoire.

Fouad Laroui tisse autour du phénomène de l'achat de riads ${ }^{1}$ par des étrangers à Marrakech l'intrigue de son roman La vieille dame du riad (Laroui, 2011). La première partie " François et Cécile », s'ouvre sur un couple de Français, désirant s'installer à Marrakech. François y investit une somme reçue en héritage " d'un oncle vieux garçon » (Laroui, 2011: 12). Ce départ ardemment souhaité par le couple ne répond ni à une curiosité culturelle ni historique. Pour Cécile, l'aventure marocaine sera l'occasion d'écrire un livre sur le pays. Aussi dès qu'elle pénètre dans le riad il lui semble voir une photo en noir et blanc avec un petit groupe d'hommes en uniforme sur un quai. Vision qu'elle décrit, toute angoissée, à François. Le registre de l'étrange va crescendo quand Cécile aperçoit dans la chambre du fond une vieille créature décrépite, présentée comme un fantôme. Aussi le couple est-il contraint de faire confiance à un jeune voisin Mansour, un professeur universitaire d'Histoire qui prétend reconstituer dans le manuscrit qu'il leur remet à lire ce que la vieille dame Massouda lui aurait raconté, sans remuer les lèvres, durant plusieurs séances nocturnes, organisées à cet effet par le couple et en sa présence. Dans son mémoire, Mansour présente au couple, transformé en lecteur, trois épisodes communs à l'Histoire du Maroc et de la France: la conférence d'Algésiras, la guerre du Rif et la deuxième guerre mondiale.

Aussi le lecteur aura-t-il un mémoire d'une centaine de pages « Histoire de Tayeb » que Mansour remet à François et Cécile, destiné à leur faire com-

\footnotetext{
${ }^{1}$ Un « riad » ou « ryhad » désigne une maison marocaine traditionnelle fermée par de hauts murs et construite autour d'un patio central dans les médinas, les centres historiques des villes.
} 
prendre la demande de Massouda qui exige, pour quitter leur riad, qu'ils lui ramènent son fils Tayeb.

Le récit de Mansour commence à la naissance de Tayeb. C'est au moment où hadj Fatmi, venu de Fès pour s'installer à Marrakech, décide d'étendre son commerce jusqu'aux confins d'Agadir. II s'y marie avec une berbère, la mère de Tayeb, morte en couches. Lalla Ghita, la première épouse arabe de hadj Fatmi, adopte le petit comme son propre fils et permet même à l'esclave Massouda de le traiter en tant que tel. Aussi, dès sa naissance, Tayeb réalise-t-il la jonction (grâce à ses trois mères) des Berbères, des Arabes et des Subsahariens.

Par la suite, François et Cécile sont transportés dans les coulisses de la conférence d'Algésiras tenue en $1906^{2}$. La fiction place hadj Fatmi au centre de l'événement historique. Cette conférence procède à la légitimation de la pénétration du Maroc par des traités internationaux. Afin de faire comprendre à François et Cécile comment le Maroc en est arrivé là, Mansour procède à un exposé très succinct sur les conditions d'intronisation de Moulay Abdelaziz ${ }^{3}$ ainsi que sur " [ses] dépenses effrénées " (M'BAREK Zaki, 2008: 109). Tableau qui sera complété quand il fait remonter la crise économique que connaît le Maroc à la prise de Tétouan par l'Espagne en 1860 et aux sommes considérables que le trésor chérifien devait lui payer comme indemnité de guerre ${ }^{4}$. Les décisions prises par la conférence ne tardent pas à nous être révélées: « L'Allemagne ${ }^{5}$ obtiendra une partie du domaine congolais de la France en 1911. L'Angleterre a les mains libres en Égypte depuis l'accord franco-anglais de 1904 " (Laroui, 2011: 110-111) et elle impose une condition que la France se verra obligée d'accepter à contrecœur, « Le nord du pays reviendra à l'Espagne " (Laroui, 2011:111) ${ }^{6}$.

\footnotetext{
${ }^{2}$ « Avec l'Acte d'Algésiras, la France et l'Espagne eurent la haute main sur les finances, l'administration et la police marocaine; autrement dit, sur les centres vitaux du pouvoir. Lié par les clauses du traité, le Sultan ne peut entreprendre aucune action dans ces domaines sans le consentement des puissances signataires de l'Acte " (M'BAREK Zaki et MIMOUN Charqi, 2008: 85).

${ }^{3}$ Moulay Abdelaziz (1878-1943), sultan du Maroc entre 1894 et 1908.

${ }^{4}$ « Si cette action de l’Europe, s'est largement déployée depuis le protectorat espagnol et français en 1912, elle s'exerçait pourtant depuis plus longtemps [...]. II faut au moins rappeler la prise d'Alger en 1830 et le premier choc survenu, quatorze ans plus tard, entre la France et le Maroc, près de l'Oued Isly. Mais l'événement qui, de l'avis des Marocains eux-mêmes ébranla sans retour leur ancien édifice, ce fut en 1859-1860, l'expédition espagnole qui aboutit à l'occupation temporaire de la ville de Tétouan [...]. Pour payer la défaite, le Maroc a versé cent dix-neuf millions, soit le revenu de ses douanes pendant vingt-cinq années. " (Germain, " Aspects de la crise financière au Maroc après l'expédition espagnole de 1860 ", Études d'Histoire marocaine, 1983: 138).

${ }^{5}$ Pour en savoir plus sur les relations du Maroc avec l'Allemagne, se reporter aux articles suivants de Germain, "La première amitié germano-marocaine (1885-1894) ", 229-247; "La crise des relations germano-marocaine (1894-1897) ", 249-291; " Sur les rapports entre l'Allemagne et le Maroc de 1870 à 1905. Un livre de Pierre Guillen », 293-305, Études d'Histoire marocaine, 1983). ${ }^{6}$ « Au bout du compte, la France finit par se frayer la voie par une série d'accords bilatéraux. Déjà le 8 avril 1904, la Grande-Bretagne lui avait laissé les mains libres au Maroc, moyennant la pareille pour elle-même en Egypte, mais moyennant aussi, l'engagement de renoncer au littoral en face de Gibraltar. En foi de quoi, il fallut bien, la même année, promettre aux Espagnols de leur aban-
} 
De retour à Marrakech, hadj Fatmi, assiste en 1912 à la signature du traité du Protectorat à Fès par Moulay Abdelhafid?

La discussion entre Hadj Fatmi et son ami Debbagh révèle à Cécile et à François, deux partis pris différents vis-à-vis de la colonisation. Si le premier, fidèle à un passéisme de mise vu sa culture religieuse prêche la guerre sainte contre l'occupant; pour le second, le pays a surtout besoin d'une modernisation que les chrétiens se chargeront de réaliser comme ils l'ont déjà fait " partout où ils se sont installés » (Laroui, 2011: 126). Mansour révèle aussi les réactions que suscite la question marocaine chez les hommes politiques français. Ce sont tous les débats houleux dans la chambre des députés entre socialistes ${ }^{8}$, communistes et conservateurs. Quant à Tayeb, il décide de rejoindre les Rifains ${ }^{9}$

donner tout le nord du pays. Quant à l'opposition allemande, la plus tenace et la plus vive, elle fut enfin levée contre cession de territoires en Afrique noire, par la reconnaissance anticipée, le 4 novembre 1911, du Protectorat de la France auquel l'Allemagne « n'opposerait aucun obstacle «. Restée maîtresse du terrain, la France, dès le 30 mars 1912, imposait au Sultan un traité qui le maintenait bien en place, mais pour servir de couverture et pour légitimer dans le même temps, l'autorité de l'étranger qui s'y substituait en fait. " (Germain, "Les implications internationales de la Guerre du Rif (1921-1926) », Études d'Histoire marocaine, 1983: 351-352).

7 " Convaincu de l'action jihadienne qui l'avait porté au trône, [Moulay Abdelhafid] se rendit bien vite compte que toute action en dépit de l'ampleur qu'elle prendrait, ne pourrait que retarder l'occupation du pays qui, sur tous les plans, face aux pouvoirs et ambitions des puissances européennes et à leurs manœuvres politiques et économiques, était en état d'être colonisé [...]. Moulay Abdelhafid perdait de plus en plus de son prestige et déçut ceux qui l'avaient porté au trône en tant que Sultan du jihad et sous condition qu'il ferait la guerre Sainte aux Français, qu'il dénoncerait l'Acte d'Algésiras et qu'il interdirait aux Européens de séjourner dans les villes de l'intérieur [...] le 30 mars 1912, l'acte du protectorat français sur le Maroc fut signé par Moulay Abdelhafid. " (M'BAREK Zaki et MIMOun Charqi, 2008: 139 et 145).

${ }^{8}$ Dans la deuxième partie du roman, intitulée "Histoire de Tayeb ", le personnage se pose la question suivante, récurrente: "Pourquoi les Français sont-ils au Maroc ? ", par trois fois car Tayeb cherche à comprendre ce qui légitime le phénomène appelé « colonisation » et à chaque fois surgit une réponse différente exprimée soit par une personnalité connue soit trouvée par le jeune homme lui-même. Pour Lyautey: " Nous avons trouvé ici un état constitué, avec son souverain, son gouvernement, ses institutions politiques, économiques et culturelles et sa diplomatie. " La seconde fois, c'est Clemenceau qui s'exprime: "N'essayons pas de revêtir la violence du nom hypocrite de civilisation. C'est la négation du droit. " Jaurès tonne: Le colonialisme est " une affaire Dreyfus permanente "; la conquête du Maroc est " une opération d'un autre âge ". Clemenceau encore: « on commence par les missionnaires, on continue par les militaires, on finit par les banquiers ". La troisième fois, Tayeb énumère d'autres raisons susceptibles de justifier l'entreprise coloniale: « L'or. Le fer. Le cuivre. Le plomb. Le zinc. Le manganèse. » (LAROUI, 2011: 128-129).

${ }^{9}$ « Les Rifains ont fait la guerre contre l’Espagne. Ils établirent une république indépendante et ils ont presque réussi à être vainqueurs dans une guerre de libération. De leur côté, les nationalistes, les citadins, se soumirent au colonialisme français sans luttes. Mais ils s'engagèrent aussitôt dans une résistance politique tout en acceptant l'Etat chérifien. Les différences sont significatives et il ne faut pas les minimiser. Chacun était une réponse à des conditions sociales déterminées. " (BRown Kenneth, "Résistance et nationalisme », Collectif, 1976: 474).

"On est toujours frappé, en lisant les archives marocaines du siècle dernier par la vigilance hostile des populations que l'on perçoit très nettement à l'arrière-plan de tous les faits de la pénétration 
dans le Nord du pays. Le héros est aux côtés d'Abd El-Krim El Khattabi ${ }^{10}$ lors d'un épisode décisif de la guerre du Rif: la bataille d'Anoual où les Rifains remportent une victoire écrasante sur les Espagnols en 192111. Il lui est donné de voir de près Abd El-Krim. Une balle blesse Tayeb au genou et il s'en retourne se faire soigner. Guéri, il revient au Rif car Abd El-Kkrim pense que, pour mieux combattre les Européens, il ne suffit pas de leur faire la guerre mais il faut aussi acquérir leur science ${ }^{12}$. Tayeb est également présent lors de l'utilisation de gaz contre ses frères rifains puisqu'il a eu « la chance d'échapper au nuage létal » (Laroui, 2011: 152). La nécessité fictive fait de lui le témoin privilégié de ce crime contre I'humanité. L'épisode du Rif se solde par la coalition franco-espagnole qui ne lésine guère sur les moyens ${ }^{13}$. Abd El-Krim se rend aux Français le 27 mai 1926.

patiente, multiforme et omniprésente des Européens. Souvent d'ailleurs, I'hostilité fait irruption jusque sur l'avant-scène quand sont pris à partie des Européens trop entreprenants et surtout les individus marocains qui acceptent de se faire leurs agents. " (Germain, " Le sentiment national dans le Maroc du XIXème siècle », Études d'Histoire marocaine, 1983: 183-184).

${ }^{10}$ Etant donné que l'orthographe du nom du héros rifain est sujette à variations selon les historiens lus, nous avons opté pour l'orthographe suivante: Abd el-Krim. « Il n'y a sans doute pas de nos jours, un autre exemple de personnage qui ait tant fait parler de lui tout en demeurant à ce point inconnu [...] son nom [...] demeure l'objet d'une méprise. Car, c'est son père, et non pas lui qui, en réalité, s'appela Abd el-Krim. " (Germain, Les Origines de la guerre du Rif, 1981: 23).

" La famille Abd el-Krim constituait le noyau du groupe ouriagheli, partisan de l'Espagne. Son père figurait sur la liste des pensionnés de la Comandancia General de Melilla [...]. À Alhucemas, Abd el-Krim reçut une instruction espagnole et arabe, aux frais du gouvernement de Madrid jusqu'à ce qu'en 1908 on lui attribue un poste au Bureau des affaires indigènes de Melilla. Il se fit remarquer par son aptitude au travail et sa fidélité à l'Espagne. C'est ce qui explique qu'en peu d'années de service on l'avait nommé successivement secrétaire adjoint de Bureau et chef du tribunal judiciaire d'appel. » (RoBerto SANCHEZ Diaz Roberto, « La pacification espagnole », Collectif, 1976: 76-77).

11 « La défaite d'Anoual en juillet 1921 et l'écroulement qui suivit de tous les postes occupés par les Espagnols, coûtèrent à l'Espagne des milliers de victimes et d'immenses pertes matérielles. " (Madariaga Maria Rosa de, « Le Parti socialiste espagnol et le Parti communiste d’Espagne face à la révolte rifaine ", Collectif, 1976: 324).

"Carleton Coon note dans son livre, Caravan: «Abd el-krim aurait pu combattre les Français pendant une période indéfinie si ses adversaires n'avaient pas utilisé contre lui, l'aviation et les blindés» [...]. Abd el-krim a été un précurseur de Mao Tsé-toung, d'Ho-Chi-Minh, de Che Guevara, dans la découverte des vertus de la guérilla. C'est cette leçon qui sera retenue par les nationalistes activistes durant les dernières phases de la lutte de libération nationale maghrébine. " (ZNIBER Mohamed, "Le rôle d'Abd el-Krim dans la lutte pour la libération nationale dans le Maghreb ", Collectif, 1976: 494).

12 «Abd el-krim fut le premier à sentir le besoin de s'organiser civilement en État: al Jumhuriya Rifiya. Dans la pratique quotidienne, il jetait les bases économiques, sociales et politiques pour une transformation du Rif. » (MADARIAGA Maria Rosa de, " Le Parti socialiste espagnol et le Parti communiste d'Espagne face à la révolte rifaine ॥, Collectif, 1976: 356-357).

« On a voulu présenter le mouvement d'Abd el-krim comme une révolte de sauvages. C'est une vieille coutume des impérialistes que de discréditer les mouvements libérateurs des peuples colonisés. Les Rifains aiment la civilisation parce qu'ils ont commencé à en connaître les bénéfices. ॥ (Ibidem, p. 350).

${ }^{13}$ « Au début du mois d'aout, l'offensive est déclenchée. Cinq cent mille hommes, soixante-dix- 
Et Tayeb de reprendre ses études en attendant le troisième événementphare retenu par Mansour pour ses deux lecteurs français: la seconde guerre mondiale dans laquelle Tayeb s'engage, à l'exemple de milliers de goumiers marocains, aux côtés de la France. Mansour peut ainsi instruire le couple de l'exploit accompli par les goumiers ${ }^{14}$ marocains lors de la deuxième guerre mondiale ${ }^{15}$.

On retrouve donc Tayeb en 1943 en Italie. II fait partie des cent mille soldats marocains qui " se distingueront particulièrement lors de la campagne d'Italie. La fameuse bataille du Monte Cassino (de janvier à mai 1944), c'est d'abord une affaire marocaine "(Laroui, 2011: 189) ${ }^{16}$ précise le récit. C'est au cours de cette bataille qu'une grenade explose et réussit à faire éclater une partie du crâne de Tayeb, emportant avec elle sa mémoire. L'image qui clôt le mémoire que lisent François et Cécile scelle à jamais le lien entre l'Histoire de la France et celle du Maroc lorsque le général de Gaulle décore le Sultan Mohammed Ben Youssef et le fait Compagnon de la Libération.

sept bataillons, trente-six compagnies de chars, seize escadrilles aériennes, le tout mené par quarante-deux généraux sous les ordres du maréchal Pétain, attaquant sur les trois cents kilomètres qui forment le front rifain. " (LAROUI, 2011:151).

"Le consensus est saisissant entre Pétain, l'état-major et les «spécialistes». La recherche d'un "succès écrasant» passe par l'emploi plus systématique et massif du matériel moderne et la mise en place d'une infrastructure routière et ferroviaire adéquate. Il faut, préconise Serrigny, «frapper chez Abd el-krim, au cœur du Rif, dans ses tribus les plus fidèles, en jetant la terreur parmi les caïds et les douars qui sont ses plus fermes appuis». " (RIVET Daniel, " Le commandement français et ses réactions vis-à-vis du mouvement rifain 1924-1926 », Collectif, 1976: 116-117).

14 « Goumier »: soldat de l'infanterie légère dans l'Armée française d'Afrique au Maroc.

15 « D’abord supplétifs, puis réguliers, les goumiers se sont surtout illustrés lors de la Seconde Guerre mondiale, au cours de laquelle les groupements de tabors (régiments), tabors (bataillons) et goums (compagnies), principalement sous les ordres du Général Guillaume, ont obtenu, entre 1942 et 1945, dix-sept citations collectives à l'ordre de l'armée et neuf à l'ordre du corps d'armée, puis en Indochine de 1946 à 1954. Le $2^{\mathrm{e}}$ groupe de tabors marocains (2 $\mathrm{CTM}$ ) est l'une des cinq unités d’infanterie les plus décorées de la Seconde Guerre mondiale ». Voir le site Wikipédia:

http://fr.wikipedia.org/wiki/Goumiers_marocains, Goumiers marocains

${ }^{16}$ Sur ce point: « En Italie, l'exploit le plus retentissant des GTM a eu lieu en mai 1944 lors de la bataille de Monte-Cassino, au cours de laquelle dix mille goumiers pénètrent dans les monts Aurunci, bastion sud de la position allemande de Monte-Cassino, «nettoient» les collines depuis le Garigliano jusqu'au sud de Rome et éliminent en trois semaines de combats certaines des unités allemandes les mieux entrainées [...]. La plupart des analystes militaires considèrent la manœuvre des goumiers comme la victoire critique qui a finalement ouvert la route de Rome aux Alliés ». Voir le site Wikipédia:

http://fr.wikipedia.org/wiki/Goumiers marocains\#ltalie 1943-1944.

Toutefois une précision s'impose dans ce cas et que nous n'avons rencontrée nulle part ailleurs au cours de nos lectures: "C'est là, au nord et au sud de Cassino, que les troupes marocaines furent engagées: en hiver au nord, puis au printemps au sud. Ici une remarque est à préciser: la légende veut que les combats pour la route de Rome aient eu lieu à Cassino. C'est vrai pour les Américains, les Polonais, les Néo-Zélandais, les Indiens et les Allemands. Mais jamais les troupes marocaines n'ont combattu à Cassino. Elles ont été engagées à 20 km au nord, pendant l'hiver 1943-1944, et à $20 \mathrm{~km}$ au sud pour la grande bataille décisive du Garigliano, le 11 mai 1944. Une fois encore la légende risquera-t-elle de l'emporter sur l'Histoire? » (Le Mémorial du Maroc 1934-1953, 1984: 120). 
Et la fiction reprend ses droits pour préciser qu'à sa mort, Lalla Ghita, la mère de Tayeb, fait promettre à Massouda de ne quitter le riad qu'après le retour de leur fils, qui « ne doit [surtout] pas trouver porte close » (Laroui, 2011: 198). La promesse est tenue lorsque Massouda demande à François et Cécile de lui rendre Tayeb pour qu'elle puisse quitter le riad qu'ils viennent d'acquérir.

Dans la troisième partie "le retour ", François et Cécile louent un appartement et s'achètent des livres sur l'Histoire du Maroc. C'est ainsi qu'ils deviennent « incollables sur les dynasties qui s'étaient succédées entre l'Atlantique et l'Atlas » (Laroui, 2011: 224). L'influence bénéfique du mémoire de Mansour donne à Cécile l'envie d'écrire autrement. Elle veut reprendre, pour la terminer, I'histoire de Tayeb. Elle veut mêler la vie de ce personnage à tous les épisodes héroïques passés et dont malheureusement, il ne peut plus se rappeler. Par son acte d'écriture, Cécile se fait la mémoire encore vivante de Tayeb, rend hommage à tous les Marocains qui ont sacrifié leur vie pour défendre la France et envers lesquels elle a un devoir de mémoire. Cet hommage scriptural prépare un hommage beaucoup plus solennel: François et Cécile font don de leur riad à la ville ocre en le transformant en un musée des tirailleurs marocains. Le jour de la cérémonie d'ouverture, la dame du riad disparaît de la chambre du fond. François a bien compris la leçon prodiguée par Mansour, Tayeb est " moins un être de chair et de sang que [le] symbole " (Laroui, 2011: 224) de toute une génération de Marocains dont le souvenir est bien incrusté dans la mémoire collective des peuples marocain et français. Quant au retour de Tayeb au riad, il est vécu aussi sur le mode symbolique puisque l'épilogue le montre sur une photo accrochée au mur de la chambre du fond, au milieu de ses compagnons d'armes sur un quai du port de Casablanca. Cécile retrouve là la vision qu'elle a eue lorsqu'elle a pénétré le riad pour la première fois: les tirailleurs marocains partis du port de Casablanca se battre aux côtés du général Guillaume ${ }^{17}$ et qui obtiendront « la Croix de guerre 1939-1945 avec trois citations à l'ordre de l'Armée, ainsi que le Mérite militaire chérifien » (Laroui, 2011: 247).

Dans ce roman la fiction et l'Histoire coopèrent amenant le lecteur, Marocain ou Français, à revisiter des tranches importantes de l'Histoire commune de leurs pays respectifs. Incapable d'opérer chacune de

\footnotetext{
17 « Officiers, sous-officiers, goumiers des $1^{\mathrm{e}}, 2^{\mathrm{e}}, 3^{\mathrm{e}}, 4^{\mathrm{e}} \mathrm{GTM}$. [...] Cette victoire éclatante, vous l'avez obstinément préparée sur tous les champs de bataille de la Libération de novembre 1942 à avril 1945, de la Tunisie au Neckar et au Danube. En Tunisie, sommairement armés et équipés, vous avez acquis par vos exploits le droit de poursuivre la lutte en Europe à l'avant-garde des Alliés. Vous avez en Sicile, vaillamment représenté l'Armée française. En Corse, vous avez libéré Bastia en attendant de prendre une part prépondérante à la conquête de l'lle d'Elbe. En Italie, votre marche foudroyante en tête du corps de montagne [...] a déconcentré l'ennemi et a ouvert la route de Rome. En France vous avez participé brillamment à la libération de Marseille, dégagé au passage Briançon, contribué [...] à chasser l'ennemi des Vosges, puis à sauver Strasbourg. » Texte de félicitations du général Guillaume adressé aux goumiers, 11 mai 1945. Voir le site Wikipédia: http://fr.wikipedia.org/wiki/Goumiers_marocains\#Hommages.
} 
son côté, afin de réaliser une telle prestation, la fiction et l'Histoire essaient toutes deux de restituer aux lecteurs contemporains des pans entiers d'une Histoire qu'ils ne penseraient peut-être pas lire sans cela. C'est sous les traits de la fiction que l'Histoire pure et dure parvient à s'imposer.

\section{Références bibliographiques}

\section{CEuvre étudiée}

LAROUI Fouad, La vieille dame du riad, Paris, Julliard, 2011.

\section{Ouvrages critiques}

Авітво Michel, Histoire du Maroc, Paris, Perrin, 2009.

AugARDE Jacques, La Longue route des tabors, Paris France Empire, 1983.

CLAYTON Anthony, Histoire de l'armée française en Afrique 1830-192I, Paris, Albin Michel, 1994.

GAUjAC Paul, « Le Corps expéditionnaire français en Italie 1943-1944 ». In : Histoire et collections, Paris, 8 janvier 2004.

GeRMAIn Ayache, Les Origines de la guerre du Rif, Paris-Rabat, Publications de la

Sorbonne (Série internationale) et Société marocaine des éditeurs réunis, 1981, tome 19.

Germain Ayache, Études d'Histoire marocaine, Rabat, Société marocaine des éditeurs réunis, Coll. Atlas, 1983.

Germain Ayache, La guerre du Rif, Paris, L'Harmattan, Coll. « Histoires et Perspectives Méditerranéennes », 1996.

LAHJOMRI Abdeljlil, Le Maroc des heures françaises, Rabat, Marsam, 1999.

Le Mémorial du Maroc 1934-1953: Lutte du Roi et du peuple. Rabat, (Collection dirigée par Larbi Essakali), Rabat, Nord Organisation copyright 1984, Volume 6.

LAROU Abdallah, Les Origines sociales et culturelles du nationalisme marocain (1830-1912), [1977], Casablanca, Centre Culturel Arabe, 2009.

M'BAREK Zaki et Mımoun Charqi, Maroc. Colonisations et Résistances 18301930, Coll. Histoire et lectures politiques, Salé, 2008.

Mounir Omar, Bou hmara. L'homme à l'ânesse, Rabat, Marsam, 2007.

MounIR Omar, Raïssouni le magnifique, Rabat, Marsam, 2012.

RIVET Daniel, Lyautey et l'institution du Protectorat français au Maroc 19121925, Tomes I. II. III, Paris, L'Harmattan, Coll. Histoire et Perspectives Méditerranéennes, Paris, 1996.

Collectif: Abd el-krim et la république du Rif, Actes du colloque international, d'études historiques et sociologiques 18-20 janvier 1973, Paris, Maspero, 1976. 


\section{Filmographie}

La Paysanne aux pieds nus, film franco-italien réalisé par Vittorio De Sica avec Jean-Paul Belmondo et Sophia Loren. Produit par Compagnia Cinematographica Champion, Titanus, Les Films Marceau, Cocinor et Société Générale de Cinématographie, 1960.

Les Goumiers marocains, film documentaire marocain d'Ahmed El Maânouni (France, $52 \mathrm{mn}, 1992$ ), France 3, Production Lille, GMT Productions.

Indigènes, film français, marocain, belge, algérien réalisé par Rachid Bouchareb avec Sami Bouajila, Rochdy Zem, Jamal Debbouze, Samy Naciri, Abdeslam Arbaoui. Produit par Teassalit Productions, en co-production avec France 2, France 3-Cinéma, Kiss Film, Studio Canal, Taza Productions, Tassili Films, Versus Production et Scope Invest, 2006.

\section{THE RIAD: FROM A PLACE OF VILLAGE TO A PLACE OF MEMORY IN THE OLD LADY OF RIAD OF FOUAD LAROUI}

François and Cécile buy a riad in Marrakech. To their astonishment, they discover Massouda who, to clear the place, demands the return of his son Tayeb. The couple seeks help from neighbor Mansour. This one gives them a memory in which the life of the son of Massouda covers a large part of the common history of Morocco and France going from the Protectorate to the Second World War.

Keywords: Fiction, History, Colonialism, Satire, Writing, Memory. 